\title{
Correction to: Weight Bearing Cone Beam Computed Tomography (WBCT) in the Foot and Ankle
}

\section{Correction to:}

\section{Richter et al., Weight Bearing Cone Beam Computed Tomography (WBCT) in the Foot and Ankle, https://doi.org/10.1007/978-3-030-31949-6}

This book was inadvertently published with the wrong chapter author details in pdf and ePub version.

In the original version of all the chapter PDFs, the name of the author was missed to be mentioned and it has been corrected now by placing the author name in the chapter opening page.

In the original ePub version, several author names were provided instead of single chapter author name which is now corrected. 